\title{
Cardiotocoğrafia Anteparto e Prog̣nóstico Perinatal em Gestaçōes Complicadas pelo Diabete: Influência do Controle Metabólico Materno
}

\author{
Antepartum Nonstress Test and Perinatal Prognosis in Pregnancies Complicated \\ by Diabetes: Influence of Maternal Metabolic Control
}

Moacyr Sanches Mascaro, Iracema de Mattos Paranhos Calderon, Roberto Antonio de Araujo Costa, Izildinha Maestá, Graziela Bossolan, Marilza Vieira Cunha Rudge

\begin{abstract}
RESUM0
Objetivo: relacionar a qualidade do controle metabólico com os resultados da cardiotocografia (CTG) anteparto e avaliar sua capacidade preditiva no prognóstico perinatal de gestações associadas ao diabete.

Pacientes e Métodos: estudo retrospectivo de 125 gestantes, portadoras de diabete gestacional ou clínico, no qual se relacionou a última CTG anteparto (intervalo máximo de 48 horas) à qualidade do controle metabólico materno e aos resultados perinatais. A qualidade do controle metabólico foi definida pela média glicêmica do dia do exame (MGd) e da gestação (MG) e pelo comportamento da requisição de insulina ( $R /$ insulina). Para os resultados perinatais foram analisados os indices de Apgar de $1^{\circ}$ e $5^{\circ}$ minuto, a classificação peso/idade gestacional, o tempo de internação, a necessidade de cuidados de UTI e a ocorrência de óbito neonatal (ONN) precoce. A capacidade diagnóstica da CTG anteparto foi avaliada pelos indices de sensibilidade, especificidade e valor preditivo positivo e negativo.

Resultados: a MGd adequada ( $<120 \mathrm{mg} / \mathrm{dL}$ ) associou-se a 2,9\% dos resultados de CTG anteparto alterados e a inadequada ( $\geq 120 \mathrm{mg} / \mathrm{dL})$, a $26,1 \%$ ( $p<0,005)$. A $M G$ mantida inadequada se relacionou a 13,7\% de CTG anteparto alterada e a adequada, a apenas $2,7 \%$ ( $p<0,005)$. O comportamento da requisição de insulina não interferiu nos resultados da CTG anteparto. Os indices de Apgar de $1^{\circ}$ e $5^{\circ}$ minuto, a necessidade de cuidados de UTI e a ocorrência de ONN precoce não dependeram do último traçado da CTG anteparto. O exame diferenciou o tempo de internação dos recém-nascidos: quando normal, 46,4\% tiveram alta hospitalar até o $3^{\circ}$ dia de vida e, quando alterado, 62,5\% deles ficaram internados por mais de sete dias.

Conclusões: os resultados alterados da última CTG anteparto relacionaram-se com níveis inadequados de $M G$, diária e da gestação, e não dependeram da $R /$ insulina. $O$ resultado normal da CTG anteparto foi adequado para garantir a saúde neonatal. Ao contrário, os resultados alterados indicaram risco de complicações nos filhos de mães diabéticas.
\end{abstract}

PALAVRAS CHAVES: Cardiotocografia. Diabete melito. Prognóstico perinatal. Monitorização fetal. Óbito neonatal.

\section{Introdução}

Diabete melito é complicação presente em 2,2 a $7,9 \%$ das gestações ${ }^{1}$. A mortalidade perinatal dos filhos de mães diabéticas decresceu nas últi-

Faculdade de Medicina de Botucatu - Unesp. Departamento de Ginecologia e Obstetrícia. Disciplina de Obstetrícia. Correspondência:

Moacyr Sanches Mascaro

Praça Sete de Setembro, 140, ap 1301 - Centro

86010-110 - Londrina - PR mas décadas, em decorrência do avanço na compreensão das adaptações metabólicas maternas, do reconhecimento da importância da manutenção da normoglicemia em gestantes portadoras de diabete e da introdução de métodos de avaliação do bemestar e da maturidade pulmonar fetais ${ }^{2}$.

O estado de descompensação metabólica materna predispõe a maior risco de hipoxemia intra-uterina, o que torna imprescindíveis o adequado controle metabólico e o diagnóstico precoce do comprometimento fetal para garantia de melho- 
res resultados perinatais ${ }^{3}$. A situação atual da assistência às mulheres portadoras de diabete pode propiciar controle metabólico, no mínimo, razoável, antes e durante a gestação. Apesar disso, o sofrimento fetal continua sendo a principal indicação de interrupção prematura destas gestações ${ }^{4}$. A avaliação do bem-estar fetal deve ser, portanto, meta prioritária nos centros de referência.

Dentro do arsenal propedêutico complementar, destacam-se as técnicas biofísicas e, em particular, a cardiotocografia sem estresse (CTG-SE) ${ }^{5}$ - o método mais comum de avaliação de bem-estar fetal, ante e intraparto, das gestações associadas ao diabete ${ }^{3}$. Este método avalia a higidez do concepto, é relacionado à função respiratória da placenta e, indiretamente, fornece indicações sobre a integridade dos mecanismos do sistema nervoso central, envolvidos no controle da freqüência cardíaca e da movimentação do feto ${ }^{6}$.

Rigg e Petrie ${ }^{7}$ recomendam que a monitorização fetal anteparto não deveria ser considerada para determinar o momento do parto, mas para permitir a decisão de que é segura a continuação da gravidez. O valor da CTG, ante e intraparto, é creditado à capacidade de identificar, com 95,0\% de acerto, o feto sadio e, conseqüentemente, a possibilidade de se prosseguir a gravidez. A CTG-SE isolada é, no entanto, método com sensibilidade de apenas 15,0 a 50,0\%, para identificar o feto comprometido $^{8}$.

São vários os protocolos de interpretação dos traçados da $\mathrm{CTG}^{6,9}$, mas a presença ou ausência de acelerações transitórias da freqüência cardíaca fetal (FCF), em resposta aos movimentos fetais, é requisito fundamental, comum a todos os protocolos de análise. A presença de acelerações transitórias em resposta a movimentos fetais confirma a boa oxigenação intra-útero e confere ao feto a qualidade de ser reativo. Estudo colaborativo nos Estados Unidos, envolvendo 18 instituições, entre 1976 e 1980, revelou aumento da morbidade perinatal, quando os fetos se mostraram não reativos na CTG-SE ${ }^{8}$.

Em nosso meio, Rudge et al. ${ }^{10}$ analisaram os traçados cardiotocográficos anteparto de 84 gestantes com diabete gestacional ou clínico, relacionados ao tipo de diabete, à média glicêmica da gestação e ao prognóstico perinatal. Os resultados da CTG-SE não dependeram da gravidade do quadro clínico materno, mas se relacionaram diretamente com o controle metabólico alcançado durante a gestação. A média glicêmica maior ou igual a $120 \mathrm{mg} / \mathrm{dL}$, considerada inadequada pelos autores, se relacionou à maior incidência de traçados anormais, identificando o comprometimento fetal.

A despeito da falta de evidências científicas, a CTG-SE ${ }^{11}$ é o teste mais utilizado para avaliar o bem-estar, nas gestações complicadas pelo diabete, e guarda relação direta com a qualidade do controle metabólico materno e com o prognóstico perinatal ${ }^{10}$. Considerando tais aspectos, seria válido o interesse em relacionar os achados da CTG-SE com parâmetros que avaliem tanto o controle metabólico como o comprometimento perinatal na população de gestantes diabéticas.

Este trabalho teve como objetivo geral relacionar a qualidade do controle glicêmico com os resultados da CTG anteparto e avaliar sua capacidade diagnóstica no resultado perinatal de gestantes portadoras de diabete gestacional ou clínico. Foram objetivos específicos determinar a influência da média glicêmica do dia do exame (MGd), da média glicêmica da gestação (MG) e do comportamento da requisição de insulina ( $\mathrm{R} /$ insulina) sobre os resultados da última CTG anteparto, e avaliar a capacidade preditiva e a relação entre este último traçado da CTG anteparto e o prognóstico perinatal.

\section{Pacientes e Métodos}

Trata-se de estudo retrospectivo, tipo coorte, no qual foram incluídas 125 gestantes portadoras de diabete gestacional ou clínico, pertencentes às classes A/B a FRH de P. White ${ }^{12}$ ou ao grupo IIB de Rudge $^{2}$, que preencheram os seguintes critérios de inclusão: uso de insulina antes da $34^{\mathrm{a}}$ semana de gestação, realização de pelo menos três perfis glicêmicos, CTG com intervalo máximo de 48 horas até o parto e parto realizado no Serviço. Foram considerados critérios de exclusão: traçado não adequado para interpretação da CTG anteparto, presença de contrações uterinas características de trabalho de parto na última CTG anteparto, gestação gemelar e ausência de registros nos prontuários das mães e recém-nascidos.

Para o cálculo do tamanho amostral considerou-se a proporção de 7\% de grávidas portadoras de diabete ${ }^{1}$, o intervalo de confiança de 95\% e a precisão de 5\%. Assim, o tamanho amostral mínimo foi fixado em 100 pacientes ${ }^{13}$.

Para o estudo da relação entre controle glicêmico e resultados da CTG anteparto, foram considerados como variáveis de controle algumas características maternas definidas por: idade materna - diferenciada em inferior e igual ou superior a 30 anos (<30 anos e $\geq 30$ anos); paridade anterior - categorizada em zero e $\geq 1$; classificação clínica - de acordo com a classificação de $\mathrm{P}$. White $^{12}$, modificada por Gabbe ${ }^{14}$ - diabete gestacional - classes A e A/B, diabete clínico de curta duração - classes B e C, diabete clínico com complicações vasculares - classes D a FRH. Como variáveis independentes considerou-se o controle 
glicêmico materno, determinado pela MGd, calculada em $\mathrm{mg} / \mathrm{dL}$, pela média aritmética dos valores de glicemia plasmática observados durante o dia da realização da última CTG anteparto ${ }^{6}$, pela MG da gestação (MG) calculada em $\mathrm{mg} / \mathrm{dL}$ pela média aritmética dos valores de glicemia plasmática de todos os perfis glicêmicos realizados durante a gestação ${ }^{15}$ e pelo comportamento da requisição de insulina, avaliada de acordo com o comportamento fisiológico e não fisiológico da R/ insulina na evolução da gestação. Para a classificação desta variável foram elaboradas curvas individuais relacionando a dose de insulina (em unidades internacionais - UI) com a idade gestacional (em semanas). Após análise individual das curvas de insulina, o comportamento da requisição de insulina foi classificado em fisiológico, quando se observou ascensão da curva entre a $24^{a}$ e a $36^{a}$ semana de gestação, e não fisiológico, quando se observou platô ou oscilação da curva entre a $24^{\mathrm{a}}$ e a $36^{\mathrm{a}}$ semana de gestação ${ }^{16}$.

As médias glicêmicas diárias e da gestação foram classificadas em adequada (MG $\leq 120 \mathrm{mg}$ / $\mathrm{dL}$ ) e inadequada (MG $>120 \mathrm{mg} / \mathrm{dL})^{15}$. Como variável dependente, considerou-se o resultado da última CTG anteparto, classificado em CTG normal (presença de, pelo menos, duas acelerações transitórias com amplitude $\geq 15$ bpm e duração mínima de 15 segundos e ausência de desacelerações) e alterado (ausência de acelerações transitórias e presença de desacelerações tardias e/ou variáveis desfavoráveis em resposta às contrações de Braxton-Hicks ${ }^{5}$.

Para o estudo da influência da CTG anteparto sobre a morbimortalidade perinatal, considerou-se como variável de controle a idade gestacional do recém-nascido (RN) e a relação peso/idade ao nascimento, definindo-se as seguinte categorias: RN pré-termo - idade gestacional inferior a 37 semanas; RN de termo - idade gestacional superior a 37 semanas; RN de peso adequado peso entre os percentis 10 e $90^{17}$; RN de peso pequeno - peso abaixo do percentil 10; RN de peso grande - peso acima do percentil 90 . Como variável independente considerou-se o traçado da última CTG anteparto. Para as variáveis dependentes foram selecionados os índices de Apgar no $1^{\circ} \mathrm{e}$ $5^{\circ}$ minuto de vida, considerando-se menor que sete $(<7)$ e igual ou superior a sete $(\geq 7)$; o intervalo, em dias, do nascimento até a alta, categorizado em menor ou igual a três $(\leq 3)$, entre quatro e sete $(4-$ 7) e maior que sete dias (>7); a necessidade de internação dos recém-nascidos em unidade de terapia intensiva (UTI) e a ocorrência de óbito fetal (natimortos) e de óbito neonatal precoce (neomortos até o $7^{\circ}$ dia de vida).

Para o estudo da capacidade preditiva da CTG anteparto no prognóstico perinatal considerou-se como teste diagnóstico o traçado da última CTG anteparto e, como padrões-ouro, os índices de Apgar de $1^{\circ}$ e $5^{\circ}$ minuto, a necessidade de UTI, o tempo de internação dos recém-nascidos e o percentual de morte perinatal.

No estudo estatístico para comparação das proporções foi utilizado o teste exato de Fischer ou o teste de Goodman, adotando-se 5\% como limite de significância estatística $(\mathrm{p}<0,05)^{18}$. Para a capacidade diagnóstica da CTG anteparto em relação ao prognóstico perinatal foram calculados os índices de sensibilidade, especificidade, valor preditivo positivo (VPP) e valor preditivo negativo (VPN).

Este projeto foi aprovado pelo Comitê de Ética em Pesquisa da FM Botucatu - Unesp.

\section{Resultados}

As 125 gestantes estavam distribuídas igualmente entre as classes A/B $(42,4 \%)$ e B-C $(42,4 \%)$. Dezenove $(15,2 \%)$ pertenciam às classes $\mathrm{D}$ a FRH de P. White ${ }^{12}$. A média de idade foi de 31 anos, correspondentes a $57,6 \%$ de gestantes com 30 anos ou mais e $42,4 \%$ com menos de 30 .

A maioria (103) delas tinha pelo menos um filho $(82,4 \%)$. O resultado normal ou alterado da CTG anteparto distribuiu-se homogeneamente nas faixas de idade materna. Os traçados normais foram característicos das multíparas $(87,1 \%)$ e os alterados foram mais freqüentes em nulíparas $(77,8 \%)$, com diferença significativa. Nenhuma gestante das classes D a FRH apresentou CTG anteparto alterada e não se observou diferença estatística nos traçados das portadoras de diabete gestacional (classe A/B) ou clínico das classes B e C (Tabela 1).

Tabela 1 - Número e porcentagem de gestantes categorizadas em faixa etária, paridade e classes de P. White, de acordo com o resultado da cardiotocografia (CTG) anteparto.

\begin{tabular}{|c|c|c|c|c|}
\hline \multirow[t]{2}{*}{ CTG anteparto } & \multicolumn{2}{|c|}{ Normal } & \multicolumn{2}{|c|}{ Alterada } \\
\hline & $\mathrm{n}$ & $\%$ & $\mathrm{n}$ & $\%$ \\
\hline \multicolumn{5}{|l|}{ Idade materna } \\
\hline$<30$ anos & 50 & 43,1 & 3 & 33,3 \\
\hline$\geq 30$ anos & 66 & 56,9 & 6 & 66,7 \\
\hline \multicolumn{5}{|l|}{ Paridade* } \\
\hline Zero & 15 & 12,9 & 7 & 77,8 \\
\hline$\geq 1$ & 101 & 87,1 & 2 & 22,2 \\
\hline \multicolumn{5}{|l|}{ Classes P. White } \\
\hline $\mathrm{A} / \mathrm{B}$ & 48 & 41,1 & 5 & 55,5 \\
\hline $\mathrm{B}$ e $\mathrm{C}$ & 49 & 42,2 & 4 & 44,5 \\
\hline $\mathrm{D}$ a $\mathrm{FRH}^{*}$ & 19 & 16,4 & - & - \\
\hline
\end{tabular}


O controle metabólico materno, avaliado pelas MGd e MG, diferenciou apenas resultados alterados da CTG anteparto. A maioria dos traçados de CTG anteparto foi normal, independentemente da MGd adequada $(97,1 \%)$ ou inadequada $(73,9 \%)$. Os traçados alterados da CTG anteparto foram mais freqüentes em presença de $M G d \geq 120$ $\mathrm{mg} / \mathrm{dL}$. No grupo de pacientes com MGd adequada, apenas 2,9\% dos resultados da CTG anteparto estavam alterados; naquelas com MGd inadequada, esta proporção atingiu 26,1\%. Essa diferença é significante (Tabela 2).

Tabela 2 - Número e porcentagem de resultados normais e alterados da cardiotocografia (CTG) anteparto, de acordo com o controle metabólico materno, avaliado pela média glicêmica (diária (MGd) e da gestação (MG)e pelo comportamento da requisição de insulina (R/Insulina).

\begin{tabular}{lcccc}
\hline MGd & \multicolumn{2}{c}{ Adequada } & \multicolumn{2}{c}{ Inadequada } \\
& $\mathrm{n}$ & $\%$ & $\mathrm{n}$ & $\%$ \\
CTG normal & 99 & 97,1 & 17 & 73,9 \\
CTG alterada* & 3 & 2,9 & 6 & 26,1 \\
MG & \multicolumn{2}{c}{ Adequada } & \multicolumn{2}{c}{ Inadequada } \\
& $\mathrm{n}$ & $\%$ & $\mathrm{n}$ & $\%$ \\
CTG normal & 72 & 97,3 & 44 & 86,3 \\
CTG alterada* & 2 & 2,7 & 7 & 13,7 \\
R/insulina & Fisiológico & Não fisiológico \\
& $\mathrm{n}$ & $\%$ & $\mathrm{n}$ & $\%$ \\
CTG normal & 86 & 92,4 & 30 & 93,75 \\
CTG alterada & 7 & 7,6 & 2 & 6,25 \\
* $\mathrm{p}<0,05$ - diferença significativa pelo teste exato de Fisher. & \multicolumn{3}{c}{}
\end{tabular}

A MG, mantida na gestação, a despeito da qualidade do controle metabólico materno, também se relacionou à maioria dos traçados normais e diferenciou os resultados anormais da CTG anteparto. Quando inadequada, esteve associada a $13,7 \%$ de CTG anteparto alterada e, quando menor que $120 \mathrm{mg} / \mathrm{dL}$, apenas $2,7 \%$ dos resultados estavam alterados. Essa diferença também foi significante (Tabela 2).

O resultado da CTG anteparto não dependeu do comportamento da R/insulina durante a gestação. A maioria dos traçados era normal, tanto na requisição fisiológica $(92,4 \%)$, como na não fisiológica $(93,75 \%)$. Entre os casos com comportamento não fisiológico, $6,2 \%$ dos traçados de CTG anteparto estavam alterados, índice estatisticamente semelhante ao observado no grupo com comportamento fisiológico da requisição de insulina $(7,6 \%)$ (Tabela 2).

As gestantes incluídas neste estudo tiveram gestação única e deram à luz 125 recém-nasci- dos, todos vivos. Nessa população, 116 traçados de CTG anteparto foram identificados como normais e nove deles estavam alterados.

Não se observou diferença significativa em relação à idade gestacional no parto e o resultado normal ou alterado da CTG anteparto. De modo contrário, quando se considerou a classificação dos recém-nascidos, o traçado normal da CTG anteparto foi mais freqüente $(71,6 \%)$ nos classificados como adequados (AIG) e o resultado alterado da CTG anteparto ocorreu em maior proporção de recémnascidos de peso grande (GIG) $(44,4 \%)$ e pequeno (PIG) $(11,2 \%)$ para a idade gestacional.

A maioria dos filhos de mães portadoras de diabete apresentou índice de Apgar $\geq 7$ no primeiro e quinto minuto de vida, independentemente do resultado da CTG anteparto. Os cuidados de UTI foram necessários em 15 e 30\% dos recém-nascidos, respectivamente, naqueles com CTG normal e alterada. O óbito neonatal ocorreu em cinco casos, correspondendo a uma taxa de morte perinatal precoce de 40/1000. Estes resultados também não foram relacionados ao último traçado da CTG anteparto (Tabela 3).

Tabela 3 - Número e porcentagem de recém-nascidos, classificados por necessidade de UTI, tempo de internação e ocorrência de óbito neonatal (ONN) precoce, de acordo com 0 resultado da cardiotocografia (CTG) anteparto.

\begin{tabular}{lrrrr}
\hline CTG anteparto & \multicolumn{2}{c}{ Normal } & \multicolumn{2}{c}{ Alterada } \\
& $\mathbf{n}$ & $\%$ & $\mathbf{n}$ & $\%$ \\
\hline Necessidade UTI & & & & \\
não & 99 & 85,3 & 6 & 66,7 \\
sim & 17 & 14,7 & 3 & 33,3 \\
Tempo internação & & & & \\
S3 dias* & 52 & 46,4 & 1 & 12,5 \\
4-7 dias & 45 & 40,2 & 2 & 25,0 \\
$>7$ dias* & 15 & 13,4 & 5 & 62,5 \\
ONN precoce & & & & \\
Não & 112 & 96,6 & 8 & 88,9 \\
Sim & 4 & 34,4 & 1 & 11,1 \\
\hline
\end{tabular}

${ }^{*} \mathrm{p}<0,05$ - diferença significativa pelo teste exato de Goodmann.

O resultado na CTG anteparto diferenciou, no entanto, o tempo de internação dos neonatos. Guando normal, $46,4 \%$ dos RNs tiveram alta até o $3^{\circ}$ dia de vida e, em presença de CTG alterada, $62,5 \%$ dos recém-nascidos permaneceram internados por mais de sete dias. Estas diferenças foram significativas (Tabela 3 ).

A análise da acurácia do último traçado de CTG anteparto revelou índices mais elevados de sensibilidade, relacionados à necessidade de cui- 
dados de UTI (66,6\%), ao tempo de internação $(88,8 \%)$ e ao risco de óbito perinatal $(88,8 \%)$. Os melhores índices de especificidade corresponderam aos resultados de Apgar de $1^{\circ}$ e $5^{\circ}$ minuto de vida: $87,9 \%$ e $96,5 \%$, respectivamente. Para qualquer resultado perinatal, a CTG anteparto demonstrou VPP baixo (5,7 a 20,0\%) e VPN elevado (80,0 a 98, 1\%).

\section{Discussão}

Sem dúvida, o controle metabólico materno parece ser o principal recurso para que a gestação complicada pelo diabete se aproxime do termo, o parto se dê por via vaginal e que o recémnascido seja saudável. Por outro lado, a segurança do bem-estar do feto permite que a gravidez se prolongue ou, pelo menos, que medidas vitais ao recém-nascido possam ser tomadas até o nascimento $^{2,15,19}$.

A média glicêmica inferior a $120 \mathrm{mg} / \mathrm{dL}$, caracterizada como adequada, não mostrou relação com os resultados da CTG anteparto. Entretanto, quando inadequada $(\geq 120 \mathrm{mg} / \mathrm{dL})$, favoreceu maior proporção de CTG anteparto alterada. Esta observação foi verdadeira, tanto para aMGd como para a MG.

Apesar de bastante variados, os dados da literatura demonstraram freqüência diminuída de resultados anormais na CTG. Dependendo dos critérios na definição do teste alterado, essa ocorrência variou entre 2,3 e $14 \%$, aumentando quando foram incluídos os resultados classificados como suspeitos ${ }^{10,19,20}$.

Neste trabalho, apenas $2,7 \%$ dos traçados foram considerados alterados. Ainda assim, a comprovação estatística de que a média glicêmica inadequada, diária ou da gestação, se relacionou a maior risco de CTG alterada é resultado que deve ser valorizado. Confirma-se, mais uma vez, a importância do controle metabólico materno estrito para garantia de melhor prognóstico perinatal ${ }^{2,15}$.

O controle metabólico inadequado favorece a hiperglicemia materna e, conseqüentemente, a hiperglicemia do meio intra-uterino, com repercussões indesejáveis sobre o feto e a placenta. Relacionaram-se à hiperglicemia materna o aumento dos depósitos de glicogênio placentário, a restrição do espaço interviloso (EIV), a hiperplasia das células da placenta no termo da gestação e a proporção aumentada de lesões vasculares, em especial, a endarterite ${ }^{21}$. Estas alterações histopatológicas caracterizam espessamento da membrana EIV/capilar fetal e confirmam dificuldade nas trocas materno-fetais, decorrentes da hiperglicemia do meio intra-uterino. A troca materno- fetal dificultada resultaria em menor oxigenação e nutrição fetais e, conseqüentemente, restrição do crescimento intra-uterino e hipóxia perinatal.

A necessidade crescente $\mathrm{R} /$ insulina evidencia comportamento fisiológico e se relaciona diretamente com o prognóstico neonatal ${ }^{16}$. Apesar disso, neste trabalho não se confirmou a influência do comportamento da R/insulina sobre os resultados da CTG anteparto.

Uma das possiveis explicações para esta nãocorrelação seria a constatação de que nem sempre a insuficiência placentária é a causa da queda na necessidade de insulina e que outros fatores podem ser envolvidos. A ingestão calórica diminuída, a falta de rigor no cumprimento dos horários, tanto para as refeições como para a aplicação de insulina, e a inconstância nas atividades diárias podem interferir nas doses requeridas de insulina. Esta multifatoriedade deve dificultar a confirmação de uma relação direta entre comportamento da necessidade de insulina e bem-estar - a queda na R/insulina poderia não ser exclusiva da insuficiência placentária e, portanto, não interferiria com a atividade biofísica fetal.

A população dos filhos de mães diabéticas foi caracterizada por maior proporção de recémnascidos AIG com resultados normais na CTG anteparto. Um número menor deles foi distribuído nas classes PIG ou GIG com resultados alterados no teste. A observação de maior proporção de traçados alterados na CTG anteparto entre os recém-nascidos de peso grande ou macrossômicos poderia ser atribuída às repercussões desfavoráveis da hiperglicemia materna sobre o meio intrauterino. A glicose materna elevada estimula o hiperinsulinismo, a eritropoese e o crescimento exagerado do feto, além de induzir a alterações placentárias que dificultam a oxigenação fetal ${ }^{21}$. A freqüência de nulíparas com significativa proporção de traçados alterados, associada ao maior risco de hipertensão gestacional ou pré-eclâmpsia, pode ter favorecido a restrição do crescimento intra-uterino e a conseqüente alteração nos traçados da CTG anteparto dos PIG.

A maioria dos recém-nascidos recebeu indice de Apgar igual ou superior a sete, tanto no $1^{\circ}$ como no $5^{\circ}$ minuto, independentemente dos resultados da CTG anteparto. Olofsson et al. ${ }^{22}$ demonstraram que a CTG, mesmo quando alterada antes do parto, se relacionou a 92 e $99 \%$ de recém-nascidos com índices de Apgar normais, respectivamente, no $1^{\circ}$ e $5^{\circ}$ minuto de vida. Estes resultados não foram diferentes dos observados neste estudo. De modo contrário, Yamamoto et al. ${ }^{19}$ observaram associação significativa entre CTG alterada e índices de Apgar diminuídos ao nascimento. Rudge et al. ${ }^{10}$, revisando traçados de CTG 
anteparto de 85 gestantes portadoras de diabete, chamaram atenção para o fato de que a CTG anteparto normal se relacionou a $20 \%$ de recém-nascidos com índices de Apgar inferior a sete, nos primeiros minutos de vida.

À semelhança dos índices de Apgar, os resultados relacionados à necessidade de cuidados de UTI neonatal e ocorrência de óbito até o sétimo dia de vida também não comprovaram influência do método de avaliação do bem-estar fetal. Estes resultados foram diferentes dos observados por alguns autores, destacando que a CTG normal evidenciou 92,3\% de recém-nascidos não admitidos em UTI neonatal ${ }^{4} \mathrm{e}$ que o exame alterado se relacionou a maior proporção de recém-nascidos com necessidade de cuidados de $\mathrm{UTI}^{19}$.

Os exames normais de CTG anteparto garantiram internação máxima de três dias à maioria dos recém-nascidos. Os traçados alterados, no entanto, identificaram maior proporção deles com alta após o sétimo dia de vida. Entretanto, dos cinco casos registrados (5/125 - 4\%), apenas um deles foi diretamente relacionado à CTG anteparto alterada.

O principal atributo da CTG sempre foi sua capacidade de garantir melhor prognóstico neonatal, em presença de traçados normais. Esta característica é válida também para as gestações complicadas pelo diabete - foram confirmados elevados índices de especificidade e de $\mathrm{VPN}^{23,24}$. Em oposição, o grande número de testes falso-positivos sinaliza que a CTG alterada raramente deve ser utilizada para a indicação do momento do parto. Nestes casos, seria necessária a complementação por outros exames, tais como a CTG com estresse (teste das contrações uterinas), o perfil biofísico fetal e a dopplervelocimetria das circulações uterino-placentárias e fetais.

Os resultados deste trabalho evidenciaram valores de especificidade de 80 a $90 \%$ para os índices de Apgar de $1^{\circ}$ e $5^{\circ}$ minuto, associados VPN elevado, entre 80 e $98 \%$, para as complicações neonatais. Confirmou-se, portanto, a principal característica da CTG anteparto, anteriormente relatada por outros autores ${ }^{23,24}$.

A morbimortalidade perinatal dos filhos de mães diabéticas pode ser relacionada a vários fatores, entre eles, controle rígido da glicemia materna, adequada avaliação ante e intraparto da vitalidade fetal, confirmação da maturidade fetal e avanços nos cuidados dos recém-nascidos. Ficaria difícil individualizar a contribuição de cada um deles e inúmeras respostas precisam ser providenciadas. A despeito da falta de evidências científicas para definir o melhor teste de avaliação fetal nestas gestações ${ }^{9,11}$, o traçado normal da CTG anteparto mostrou-se adequado para garantir a saúde neonatal dos filhos de mães diabéticas.

\section{SUMMARY}

Background: the antepartum nonstress test (NST) is the most commonly used test to evaluate fetal well-being in pregnancies complicated by diabetes, its results being related to the quality of maternal metabolic control and perinatal prognosis.

Purpose: to relate the quality of metabolic control to the results of the NST and to evaluate its predictive capacity for the perinatal prognosis of pregnancies associated with diabetes.

Patients and Methods: this is a retrospective study of 125 pregnant women with gestational or clinical diabetes in which the last NST (maximum interval of $48 \mathrm{~h}$ ) was related to the quality of maternal metabolic control and perinatal results. Quality of metabolic control was defined by the glycemic mean on the test day (GMd), glycemic mean during pregnancy $(G M)$, and behavior of insulin requirement (Insulin/R). For the perinatal results, the following parameters were evaluated: the 1st and 5th min Apgar scores, the gestational weight/age classification, the length of hospitalization, the use of neonatal ICU, and the occurrence of early neonatal death. Diagnostic capacity of the NST in relation to the perinatal results was evaluated by sensitivity and specificity values, positive predictive value, and negative predictive value.

Results: the adequate GMd $(<120 \mathrm{mg} / \mathrm{dL})$ on the test day showed that $2.9 \%$ of the NST results were abnormal; for inadequate $G M(\geq 120 \mathrm{mg} / \mathrm{dL}), 26.1 \%$ ( $p<0.005)$. Maintained inadequate GM during pregnancy was related to $13.7 \%$ abnormal NST; that adequate to only $2.7 \%$ ( $p<0.005)$. Insulin requirement behavior did not interfere with the NST. The 1st and 5th min Apgar scores, use of ICU, and occurrence of neonatal death did not depend on the last NST result. This test influenced the length of newborn hospitalization: when normal, $46.4 \%$ were discharged on up to the $3 r d$ day after birth; when abnormal, $62.5 \%$ were discharged after the 7 th day of birth.

Conclusions: the abnormal results of the last antepartum NST correlated with inadequate MG levels (daily and during pregnancy) and did not depend on insulin/R. Normal NST was effective to ensure neonatal health. In contrast, abnormal results were related to longer hospitalization and risk for complications in diabetic mothers' newborns.

KEY WORDS: Antepartum non-stress test. Diabetes. Metabolic control. Perinatal prognosis.

\section{Agradecimento}

À Professora Doutora Lídia Raquel de Carvalho, do Departamento de Bioestatística do Instituto de Biociências da Universidade Estadual PaulistaUnesp, pela colaboração na análise estatística dos resultados. 


\section{Referências}

1. Fadel HE, Hammond SD. Diabetes mellitus and pregnancy: management and results. J Reprod Med 1982; 27:56-66.

2. Rudge MVC, Calderon IMP, Ramos MD, Maestá I, Souza LMS, Peraçoli JC. Perspectiva perinatal decorrente do rígido controle pré-natal em gestações complicadas pelo diabete. Rev Bras Ginecol Obstet 1995; 17:26-32.

3. Landon MB, Gabbe SG, Sachs L. Management of diabetes mellitus and pregnancy: a survey of obstetricians and maternal-fetal specialists. Obstet Gynecol 1990; 75:635-40.

4. Landon MB, Gabbe SG. Fetal surveillance and timing of delivery in pregnancy complicated by diabetes mellitus. Obstet Gynecol Clin North Am 1996; 23:109-23.

5. Mariani Neto C. Comparação entre a análise visual e a computadorizada de registros cardiotocográficos anteparto em gestações de alto risco [tese]. Campinas: Universidade de Campinas; 1999.

6. Zugaib M, Behle I. Fisiopatologia cardiovascular aplicada à monitorização fetal. In: Behle I, Zugaib M, editors. Monitorização Fetal Eletrônica. $1^{\text {a }}$ ed. São Paulo: Roca; 1981. p.?

7. Rigg IA, Petrie RH. Fetal biochemical and biophysical assesment. In: Reece EA, Constan DR, editors. Diabetes mellitus in pregnancy. Principles and practice. $1^{\text {st }}$ ed. New York: Churchill Livingstone; 1988. p.?

8. Freedman RK, Anderson G, Dorchester W. A prospective multi-institutional study of antepartum fetal heart rate monitoring. I Risk of perinatal mortality and morbidity according to antepartum fetal heart rate test results. Am J Obstet Gynecol 1982; 143:771-7.

9. American College of Obstetricians and Gynecologists practice bulletin. Antepartum fetal surveillance. Number 9, October 1999. Clinical management guidelines for obstetrician-gynecologists. Int $\mathrm{J}$ Gynaecol Obstet 2000; 68:175-85.

10.Rudge MVC, Calderon IMP, Ramos MD, Suetaki H, Sá ACJ, Cruz MA. Avaliação da vitalidade dos fetos de gestantes diabéticas: análise cardiotocográfica. Rev Ginecol Obstet 1994; 5:201-7.

11.Pattison N, McCowan L. Cardiotocography for antepartum fetal assessment. Cochrane Database Syst Rev 2000; (2):CD001068.

12.White P. Pregnancy complicating diabetes. Am J Med 1949; 7:609-16.
13. Fonseca JS, Martins GA. Amostragem. In: Fonseca JS, Martins GA, editores. Curso de estatística. $6^{\text {a }}$ ed. São Paulo: Atlas; 1996. p.77-85.

14. Gabbe SG. Management of diabetes mellitus in pregnancy. Am J Obstet Gynecol 1985; 153:824-8.

15. Rudge MVC, Calderon IMP, Ramos MD, Abbade JF, Rugolo LM. Perinatal outcome of pregnancies complicated by diabetes and by maternal daily hyperglycemia not related to diabetes. A retrospective 10-year analysis. Gynecol Obstet Invest 2000; 50:108-12.

16. Migiolaro H. O comportamento da necessidade de insulina em gestantes diabéticas. Influência no controle da glicemia materna e no resultado perinatal [dissertação]. Botucatu: Universidade Estadual Paulista "Júlio de Mesquita Filho"; 2000.

17.Lubchenco LO, Hansman C, Boyd E, Dressler M. Intrauterine growth as estimated from liveborn birth-weight data at 24 to 42 weeks of gestation. Pediatrics 1963; 32:793-800.

18.Jekel JF, Elmore JG, Katz DL. Análise bivariada. In: Jekel JF, Elmore JG, Katz DL, editores. Epidemiologia, Bioestatistica e Medicina Preventiva. $1^{\text {a }}$ ed. Porto Alegre: Artmed; 1999. p.155-77.

19.Yamamoto M, Francisco RPV, Miyadarira S, Banduki Neto JD, Zugaib M. Avaliação da vitalidade fetal em gestantes diabéticas: análise dos resultados neonatais. Rev Bras Ginecol Obstet 2000; 22:55766.

20.Pereira BG, Faúndes A, Parpinelli MA, Chinaglia MLM. Avaliação da capacidade preditiva para Apgar, sofrimento fetal e anóxia neonatal da cardiotocografia anteparto e da percepção materna dos momentos fetais em gestantes diabéticas com e sem hipertensão. Rev Bras Ginecol Obstet 1997; 19 Supl:62.

21. Calderon IMP, Rudge MVC, Ramos MD, Peraçoli JC. Estudo longitudinal, bioquímico e histoquímico de placentas de ratas diabéticas: relação com a macrossomia e o retardo de crescimento intrauterino. Rev Bras Ginecol Obstet 1999; 21:91-8.

22. Olofsson P, Sjoberg NO, Solum T. Fetal surveillance in diabetic pregnancy. I. Predictive value of the nonstress test. Acta Obstet Gynecol Scand 1986; 65:241-6.

23.Pereira BG. Diabetes: assistência In: Neme B, editor. Obstetrícia Básica. $2^{\text {a }}$ ed. São Paulo: Sarvier; 2000. p.389-97.

24.Alfirevic Z, Neilson JP. Biophysical profile for fetal assessment in high risk pregnancies. Cochrane Database Syst Rev 2000; (2):CD000038. 